\title{
Las rogativas públicas en Oviedo $(1550-1840)$
}

'Uno de los campos de trabajo más recientes para el historiador es el de las mentalidades, comportamientos y actitudes colectivas, o como se le quiera llamar. La influencia que en esta historia han tenido los planteamientos y métodos de otras disciplinas como la antropología y la sociología son innegables; aunque los puntos de vista del historiador y del antropólogo sean en ocasiones muy distintos ${ }^{1}$. De estas disciplinas, la historia ha tomado y hecho suyo el interés por las manifestaciones sociales de la vida cotidiana, de las cuales se pueden inferir - con mayor o menor acierto- los soportes ideológicos y mentales de los grupos humanos en el pasado, así como sus variaciones en el tiempo. Las fiestas, las ceremonias religiosas, las cofradías, los gremios, las diversiones, las comidas, los hábitos de lectura, etc., se constituyen en otros tantos objetivos de investigación, de los que pueden extraerse elementos muy interesantes para la comprensión del pasado y también del presente. Entre estos objetos de investigación se encuentran las rogativas públicas, todavía poco analizadas desde esta perspectiva histórica.

El estudio de las rogativas suscita, inevitablemente, un problema conceptual previo: el de si se puede hablar con propiedad de una religión popular, o de modo más genérico, de una cultura popular, distinta de una cultura elitista. Se suele atribuir lo popular a la masa indiferenciada e iletrada, y lo elitista a la nobleza y el clero, sectores sociales minoritarios, capaces de acceder a una cultura escrita. En el caso de la religiosidad, la distinción suele hacerse entre la religión de los laicos y la de los eclesiásticos; la una espontánea y vital, la otra oficial, más rígida y formalista y con tendencia a imponerse sobre la anterior.

Estas diferencias tienen mucho de artificial; las investigaciones históricas y sociológicas ponen de manifiesto que no existe esa comparti-

1 Ver por ejemplo lo que señala Andrejs Plakans, Kinship in the past. An anthropology of European family life, 1500-1900 (Oxford: Basil Blackwell Publisher, 1984), pp. 248-252. 
mentación estricta que incomunica sectores sociales y hábitos culturales hasta llegar a una oposición entre «lo popular» y «lo elitista». Por el contrario, lo que se da es una transferencia de contenidos en ambos sentidos, una coincidencia en parte de las actitudes y comportamientos ${ }^{2}$.

En este estado de cosas, nos parece que la distinción entre formas culturales no debe establecerse sobre bases socioeconómicas, pues, como hemos señalado, resulta engañosa ${ }^{3}$. Nos parece más adecuada y útil la denominación que Lauwers propone de «cultura folklórica» para referirse a aquellas formas culturales cuyo contenido no es el resultado de una elaboración intelectual específica, sino que son una respuesta a necesidades en cierto modo más inmediatas; están ligadas a un movimiento espontáneo de sus protagonistas y su continuidad es fruto del respeto a la tradición recibida y asumida (aunque la tradición no agota el contenido de esta cultura folklórica) ${ }^{4}$. No se trata de algo exclusivo del "pueblo», sino que también aristócratas y eclesiásticos participan en su elaboración y transmisión.

El concepto es impreciso y su denominación tal vez no sea afortunada. Pero tiene al menos la ventaja de eliminar el envaramiento que origina la contraposición artificiosa de lo popular y lo elitista y se introduce en esos terrenos fronterizos entre formas socioculturales, en los que son más frecuentes los intercambios que las oposiciones's. En el campo concreto que nos ocupa, el de la religiosidad, el uso de este concepto supone no sólo aumentar el campo de pertinencia, sino superar

2 Sobre la insuficiencia de las sistematizaciones propuestas para la sociología de la cultura popular, ver Zev BARBU, «La cultura popular: un enfoque sociológico», Examen de la cultura popular (México: Fondo de Cultura Económica, 1982), pp. 64 y ss. Al hablar de la religiosidad popular, Chartier señala que ésta no debe considerarse como radicalmente distinta de la de los clérigos, ni como totalmente modelada por éstos. Propone sustituir la consideración de conjuntos sociales "puros», por otra que considere cada forma cultural como la unión de elementos sólidamente incorporados por sus protagonistas, elementos cuyo origen no tiene necesariamente que ser el mismo. Ver Roger CHARTIER, "La culture populaire en question», Histoire, 8 (1981), pp. 85-96.

3 «Given the flexibility of popular culture in appropiating and adapting models which it was hoped simply to impose on it from above, we may have to abandon altogether the search for cultural forms exclusive to especific groups and concentrated instead on the category of use». Stuart CLARK, "French historians and early modern popular culture», Past and Present, 100 (1983), p. 63.

4 Ver M. LAUWERS, «Religion populaire, culture folklorique, mentalités. Notes pour une anthropologie culturelle du moyen âge», Revue d'Histoire Ecclésiastique, 2 (1987), pp. 227 y ss.

5 «Les types de culture ne sont pas totalement étrangers l'un à l'autre. Les 'surfaces de recouvrement' sont nombreuses» (M. LAUWERS, op. cit., p. 247). 
esa visión de la religiosidad popular como algo limitado a las supersticiones y comportamientos anómalos.

La necesidad de esta revisión conceptual queda de manifiesto al estudiar las rogativas públicas, pues se resisten a su inclusión en rígidas categorías socioeconómicas. Como veremos, no son sólo de iniciativa popular, ni sólo participa el pueblo; desde la corona hasta los sectores sociales más inferiores, todos tienen algo que decir y hacer, y no siempre dicen y hacen lo mismo.

\section{LOS MOTIVOS DE CONVOCATORIA DE LAS ROGATIVAS}

Desde el punto de vista histórico y sociológico, las rogativas constituyen una notable fuente de información sobre variados aspectos de una sociedad; en primer lugar, sobre sus valores religiosos, evidentemente. Su estudio, junto con el de otras prácticas individuales y colectivas, permite aproximarnos al nivel de asimilación de los contenidos doctrinales, a las devociones, ritos y cultos particulares, etc.

Pero su interés se extiende a otros componentes de los hábitos sociales. Las rogativas reflejan los temores y angustias cotidianos de una sociedad agraria (nuestro campo de trabajo es el Antiguo Régimen), con una economía de subsistencia muy dependiente de las cosechas y por tanto de las condiciones climáticas ${ }^{6}$; de una sociedad que carece de recursos humanos, científicos y técnicos para enfrentarse con las enfermedades y controlar las epidemias. $Y$ son también un medio para fomentar la cohesión social en torno a la corona y los problemas del Estado, mediante la convocatoria de estas ceremonias en determinadas circunstancias.

Los trabajos sobre esta materia son todavía escasos en España. En las páginas que siguen describiremos los motivos y algunas circunstancias de una pequeña muestra de las rogativas celebradas en la ciudad de Oviedo entre los años 1550 y 1840 . La fuente de información la constituyen los acuerdos capitulares del cabildo catedralicio y del ayuntamiento ovetense.

6 Escribe el doctor Casal a mediados del siglo xvill que con los calores del verano «nos vemos precisados a pedir a Dios, con rogativas públicas, el socorro de la agua, para que no se pierda el maíz, que es el mantenimiento ordinario de los labradores»; Gaspar CaSal, Historia natural y médica de el Principado de Asturias (Oviedo: Escuela Tipográfica del Hospicio, 1900) (edic. fac. Diputación Provincial de Oviedo: Oviedo, 1959), p. 91. 
En total hemos recogido información de 146 ceremonias de este tipo, que hemos distribuido en cinco grupos según los motivos de su convocatoria. En el primero incluimos las rogativas celebradas por alguna necesidad climática; en el segundo, las celebradas por pestes y otros procesos epidémicos; el tercero agrupa las peticiones hechas por sucesos políticos, tanto internos como externos, y otros de carácter general 7 ; en el cuarto se incluyen las rogativas por miembros de la familia real; y en el último, bajo el título de «otras», hemos reunido a las que no son clasificables en ninguno de los grupos anteriores ${ }^{8}$. La distribución queda resumida en la siguiente tabla:

\begin{tabular}{|c|c|c|c|c|c|c|}
\hline & $\begin{array}{c}\text { I } \\
\text { Tiempo }\end{array}$ & $\begin{array}{c}\text { II } \\
\text { Epidemias }\end{array}$ & $\begin{array}{c}\text { III } \\
\text { Sucesos } \\
\text { políticos }\end{array}$ & $\begin{array}{c}\text { IV } \\
\text { Familia } \\
\text { real }\end{array}$ & $\begin{array}{c}\mathrm{V} \\
\text { Otras }\end{array}$ & Total \\
\hline s. XVI (2.'mitad) & 9 & 9 & 6 & 5 & 1 & 30 \\
\hline XVII & 23 & 9 & 13 & 7 & 2 & 54 \\
\hline XVIII & $9^{\circ}$ & 3 & 7 & 10 & 5 & 34 \\
\hline Total ........ & $41(35 \%)$ & $21(18 \%)$ & $26(22 \%)$ & $22(19 \%)$ & $8(6 \%)$ & 118 \\
\hline s. XIX $(1$. mitad $)$ & 8 & 7 & 4 & 8 & 1 & 28 \\
\hline Total ........ & $49(34 \%)$ & $28(19 \%)$ & $30(20 \%)$ & $30(20 \%)$ & $9(6 \%)$ & 146 \\
\hline
\end{tabular}

La proporción mayor corresponde a las rogativas celebradas por los «buenos.temporales» y para que remitan las epidemias; entre los siglos XVI y XVIII estas rogativas suponen el $53 \%$ de las celebradas en Oviedo, al menos según la muestra que empleamos. No se observa variación alguna en la primera mitad del siglo XIX; de las 28 que conocemos, 15 corresponden a las finalidades antes señaladas, es decir, el $53 \%$. Este porcentaje es similar, aunque algo inferior, al obtenido en Barcelona;

7 El 5 de octubre de 1629 «tubo noticia el cabildo que Su Magestad ha ordenado que las yglesias destos reynos agan plegarias públicas[...] por el buen suceso de la flota y armada que se espera de las Yndias», rogativa que acuerda realizar (Archivo Capitular de Oviedo, libro de acuerdos n." 23, f. 474 vto.). Más interesante es el acuerdo del 15 de noviembre de 1568 para acceder a la petición de Felipe II y hacer unas rogativas «por el socorro y favor y necesydad en que está el duque de Alva en las guerras contra luteranos» (ACO, libro de acuerdos n. ${ }^{\circ} 11, \mathrm{f} .108$ vto.).

8 Como por ejemplo la procesión que, por acuerdo tomado el 23 de octubre de 1584, se celebra con las reliquias de Santa Eulalia para que les envíe kun buen prelado» (ACO, libro de acuerdos n." 18, f. 61 vto.); el 13 de abril de 1730 se establecen los detalles para la rogativa "para el acierto y buena elección de Papa» (ACO, libro de acuerdos n." 46 , f. 285 vto.). 
para los años 1550-1620, Isabel Lobato cifra la proporción en un 59\% 9 mientras que el resultado al que llega Magdalena Mirabet en el XVIII es ligeramente superior, un $62 \%{ }^{10}$.

Las repercusiones más directas e inmediatas que tienen sobre la población los fenómenos naturales y epidémicos - sequías, lluvias torrenciales, pestes - proporcionan sobrada explicación al predominio de las rogativas de los dos primeros grupos. La cercanía de estos hechos dan como resultado una mayor iniciativa y una multiplicación de los actos; por el contrario, ante situaciónes más remotas - como la proximidad del parto de la reina - apenas si se reiteran las rogativas, salvo en el caso de que insista la autoridad convocante.

\section{LAS INICIATIVAS DE LAS AUTORIDADES CIVILES}

En la documentación analizada, la orden y petición de rogativas llega al cabildo de la catedral (que es el encargado de organizarlas para la ciudad) bien de la autoridad municipal, bien de la real. En otras ocasiones, como más adelante señalaremos, es el propio cabildo el que propone efectuar algunas rogativas. Veamos algunos ejemplos y situaciones que rodean a las convocadas por las autoridades civiles.

El 5 de julio de 1577 el ayuntamiento de Oviedo acuerda tratar con el cabildo que se haga una procesión de rogativas con todas las cofradías y conventors de la ciudad a fin de preservarla de la epidemia de peste. A finales de agosto del mismo año, se acuerda celebrar más procesiones e invitar a ellas al cabildo y a los monasterios de religiosos y religiosas, además de levantar una ermita a San Sebastián ${ }^{11}$.

El 30 de agosto de 1652 una comisión del ayuntamiento se presenta en la reunión del cabildo para pedir que se hagan rogativas a San Roque para prevenir el posible contagio de la peste que en aquellos momentos aquejaba a Aragón ${ }^{12}$.

9 Ver Isabel LoBATO I FrANCO, «Religió i societat: processions i rogatives públiques a Barcelona, 1550-1620», Primer Congrés d'Història moderna de Catalunya (Barcelona: Universidad de Barcelona, 1984), II, p. 432. En estos años el $49 \%$ de las rogativas obedecen a la falta de lluvias, y el $10 \%$ a diversos brotes epidémicos.

10 Ver Magdalena Mirabet i CuCAlA, «Pregàries públiques a la Barcelona del segle xVIII», en ibid., p. 488. Sus resultados son: lluvias, 34,5\%; buen tiempo, 22,4\%; terremotos, $2,6 \%$; pestes, $1,3 \%$; plagas, $0,8 \%$. El total asciende a $61,6 \%$.

11 Ver Ciriaco Miguel VIGIL, Colección histórico-diplomática del Ayuntamiento de Oviedo (Oviedo: Imprenta de Pardo, Gusano y Cía., 1889), pp. 447-448.

12 Ver ACO, libro de acuerdos n." 26, f. 287 vto. 
Unos cuantos años después, el 12 de noviembre de 1666,

entraron los señores comisarios de la Ciudad y en su nombre significaron el daño que esperimentava y esperava si prosiguía el tiempo con tantas aguas, por no dar lugar a la sementera; y para que ésta se hiziese y el tiempo serenase, suplicando intercediese con nuestra patrona Santa Eulalia para que, aviendo la rogativa ordinaria, continuase en semejantes aprietos sus continuados milagros ${ }^{13}$.

Peticiones como las anteriores se repiten con frecuencia entre los siglos XVI y XVIII, y continúan también durante la primera mitad del XIX ${ }^{14}$. Con estos ejemplos, queda claro el papel jugado por las autoridades municipales en estas ceremonias.

También desde la corona se promueven algunas rogativas. El 1 de marzo de 1588 se lee en el cabildo de la catedral una cédula real en la que se solicitan rogativas y oraciones "por el buen subçeso de la armada que se haçe contra ynfieles» ${ }^{15}$; tras la lectura, se toman los acuerdos pertinentes. Pocos meses después, el 15 de julio de 1588, se recuerda que deben continuarse las procesiones y rogativas «por los buenos subçesos de la armada que el Rei nuestro señor haçe contra luteranos de Yngalaterra» ${ }^{16}$; y lo mismo el día 19. Lo que ya no se recoge en las actas capitulares son los ecos del desastre sufrido por la Armada Invencible en los días siguientes.

Acontecimientos relevantes del siglo XVII, como la revuelta catalana y la guerra con Portugal, también hacen acto de presencia en la vida cotidiana asturiana por medio de las rogativas públicas. A comienzos de abril de 1642 se lee en la reunión del cabildo una carta del rey en la que señala «los aprietos en que se halla su reyno con los rebelados del principado de Cataluña y reyno de Portugal» ${ }^{17}$; el cabildo de la catedral acuerda hacer una procesión y misa solemne con sermón para los fieles, a cuyos actos se invitará al gobernador del Principado y otras autoridades. Más adelante, en septiembre de 1650, en respuesta a una petición del presidente del Consejo de Castilla, se acuerda tratar con el

13 ACO, libro de acuerdos n." 28, f. 374.

14 En abril de 1817 se solicitan para remediar la escasez de agua (ver ACO, libro de acuerdos n." 68, fs. 38 y vto.); lo mismo a mediados de siglo, entre 1846 y 1847 (ver ACO, libro de acuerdos n." 72, fs. 296 y vto., y 337 y vto.).

15 ACO, libro de acuerdos n." 19, f. 319 vto.

16 Ibid., f. 347 vto.

17 ACO, libro de acuerdos n." 25, f. 92 vto. 
obispo lo que se ha de hacer "por el buen suceso de la entrada del exército en Cataluña» ${ }^{18}$.

Sobre la guerra con Portugal, además de la mención anterior, hemos encontrado otras dos motivadas por sendas cartas de Felipe IV. Una corresponde al 30 de julio de 1661, poco tiempo después de la alianza hecha por Portugal con Inglaterra; el cabildo dispone

una rogativa pública con una misa cantada y procesión por las calles por donde ba la de la octaba del Corpus, y se avise a la Ciudad en la forma acostumbrada, y se saque la Cruz de la Victoria, y después, hasta saver el fin, suceso de lo que obrare el exército, mientras estubiere dentro de Portugal se diga cada día una misa reçada en la Cámara Santa ${ }^{19}$.

La otra mención es del 1 de junio de $1663^{20}$.

La Guerra de Sucesión, con la que se inicia el siglo XVIII, también da lugar a la celebración de rogativas; al igual que las anteriores, la iniciativa parte de la corona. El 5 de mayo de 1702 se lee en el cabildo una carta del rey (Felipe V) en la que «encarga que con prontas y continuas rogativas se pida y suplique a Dios nuestro Señor se sirva de asistirle en todas sus operaciones» ${ }^{21}$. Los miembros del cabildo acordaron celebrar tales rogativas con misa solemne y procesión, acuerdo que se transmite al ayuntamiento para que éste lo haga público. Las celebraciones se suceden con relativa frecuencia; el 2 de abril de 1704

acordaron sus mercedes [los miembros del cabildo] que mañana juebes se concluya la rogatiba de novenario que se está haciendo para la conservación de la salud de nuestro rey y señor, que Dios guarde, y por los buenos sucesos de la campaña [...], y para ello se vaya al convento de Santo Domingo con procesión general, levando la ymagen de Nuestra Señora del Rey Casto y el cuerpo de Santa Eulalia de Mérida [...] con la Cruz de la Victoria ${ }^{22}$.

Es de suponer que las rogativas continuarían hasta la firma del tratado de Utrech.

Los acontecimientos de la familia real - bodas, partos, matrimonios, defunciones - ocupan una parte importante de la muestra, entre un 19\%

18 ACO, libro de acuerdos n." 26, f. 185 vto. En julio del año siguiente, el Consejo reitera sus peticiones; ver ibid., f. 228.

19 ACO, libro de acuerdos n." 28, f. 28.

20 Ver ibid., fs. 147 vto. y 149 vto.

21 ACO, libro de acuerdos n." 38, f. 43 vto.

22 Ibid., f. 189 vto. 
y un $20 \%$, como puede verse en la tabla. La iniciativa para tales rogativas parte también de la corona ${ }^{23}$.

No queremos terminar este apartado sin señalar dos rogativas peculiares en su finalidad: la solicitada en 1631 por la aplicación de un nuevo tributo sobre la sal ${ }^{24}$; y las rogativas generales que Felipe V pide en 1727 "por la espulsión de los ministros evangélicos del Reyno de la China, para que Dios nuestro Señor mire con ojos de su divina piedad este lastimoso caso» ${ }^{25}$.

\section{LAS INICIATIVAS ECLESIÁSTICAS}

El objetivo de las rogativas que proponen las autoridades eclesiásticas depende del ámbito jurisdiccional de éstas. Mientras que el cabildo catedralicio se limita habitualmente a los sucesos locales, las disposiciones llegadas de Roma se refieren a problemas que aunque de modo inmediato repercuten en la sede romana, sus consecuencias son más amplias. Señalaremos tres ejemplos de este último caso, antes de pasar a describir la iniciativa capitular.

A mediados de enero de 1607 se da cuenta en la reunión del cabildo «cómo Su Santidad Paulo V, por las neçesidades de la Iglesia y rebelión de Veneçia» había dado orden a uno de sus cardenales que escribiese a los arzobispos españoles para que se organizasen rogativas por tales intenciones. Esta petición llega a la sede ovetense a través del arzobispo de Santiago. El cabildo nombra dos representantes para que de acuerdo con el obispo «ordenen lo que se aya de haçer para cumplir con esta obligación» 26 .

Casi cuarenta años después, el 2 de octubre de 1645, se lee una carta de Inocencio $\mathrm{X}$; el resumen del acta capitular es suficientemente expresivo:

Da quenta del aprieto en que se alla la christiandad y las guerras tan sangrientas entre los príncipes christianos, orixinado todo de los vicios y pecados que contra

23 Ciriaco Miguel Vigil recoge bastantes de las solicitadas y celebradas por estos motivos en op. cit., pp. 522-544.

24 «Para que nuestro Señor se sirba disponer como más combenga a su santo serbicio la ymposición de nuevo tributo de la sal que se pueso en lugar de los millones del vino, aceyte y carne» (ACO, libro de acuerdos, n." 23, f. 581).

25 ACO, libro de acuerdos n." 46, f. 60.

26 ACO, libro de acuerdios n." 21, f. 380 
su Divina Magestad cada ynstante están cometiendo los pecadores, olvidados $[\ldots]$ de las obligaciones de fieles christianos; por lo qual y otras muchas raçones que dicha carta refiere, Su Santidad se determinó escrivir a toda la christiandad representando este triste y lamentable estado, encargando a los prelados en sus diócesis procuren castigar los vicios y pecados públicos, y en todas partes se agan grandes demonstraçiones de penitenzias públicas y se agan rogativas para que por medio de ellas nuestro Señor se sirba de enbaynar la espada de su rigor, y husando de su misericordia nos dé buena paz y concordia entre los príncipes christianos y vitoria contra los ynfieles enemigos de su sancta fe cathólica.

Se acordó hacer «lo más presto que se pueda $[\ldots]$ plegarias públicas por la edificazión del pueblo» ${ }^{27}$.

En la misma línea que en el caso anterior, aunque con circunstancias diferentes, está la carta que se recibe del nuncio el 24 de marzo de 1703. Solicita que se hagan rogativas por los temblores de tierra que recientemente había sufrido Roma, y por la inseguridad que para la ciudad suponían «las muchas guerras de toda la Europa»; indica que se debe exhortar al pueblo para que sea conocedor de estos hechos ${ }^{28}$.

En lo tocante al cabildo de la catedral de Oviedo, lo visto hasta el momento se refiere a su actuación como organizador de las rogativas promovidas por otras instituciones. Pero tuvo también un papel realmente importante como promotor de estos actos religiosos públicos. Al igual que el ayuntamiento, el motivo de la mayor parte de sus convocatorias son por situaciones que afectan más directamente al Principado y a su capital; las menos tienen motivos de índole general ${ }^{29}$.

La falta o exceso de lluvia ocupan gran parte de los acuerdos sobre plegarias públicas. El 20 de marzo de 1586 «tratóse que el temporal del tiempo porfían mucho las aguas e los frutos se pierden. Determinóse que se hagan proçisiones e se saquen los cuerpos santos, suplicando a nuestro Señor por el buen temporal» ${ }^{30}$. También "por el temporal» se acuerda celebrar rogativas el 5 de junio de 1589; pero ahora se añade otro motivo, la seguridad física del litoral asturiano: «suplicar a nuestro

27 ACO, libro de acuerdos n." 25, f. 347. En 1648, con la paz de Westfalia, terminará la Guerra de los Treinta Años.

28 Ver ACO, libro de acuerdos n." 38, f. 107 vto.

29 El 1 de junio de 1587 se acuerda hacer una procesión de rogativa por la salud del rey, «pues es cosa que tanto ynporta»; a la misa solemne y procesión se convoca al pueblo y a las cofradías "para que bengan [...] con gran deboçión ha haçer la dicha rogativa y encomendarle (al rey) a Dios" (ACO, libro de acuerdos, n." 19, f. 258).

sô ACO, libro de acuerdos n." 18, f. 167 vto. 
Señor no permita que la armada enemiga haga daño en esta costa como hiço en la Galiçia, y pretende haçer» ${ }^{31}$.

Las rogativas para que mejore el tiempo, o en demanda de lluvia, se suceden en los siglos estudiados. Junto con ellas, aparecen también funciones religiosas en acción de gracias por los beneficios recibidos. Así, el 7 de febrero de 1605

trataron sus mercedes de que ayer, en sacando el cuerpo santo de la gloriosa Santa Eulalia, fue nuestro Señor servido de darnos agua, y que es raçón acudirle con graçias. $\mathrm{Y}$ así acordaron que le digan nueve misas en el altar mayor en nueve días, y la novena con mucha solemnidad, con que se buelva a la Cámara Santa 32.

Las pestes y otras epidemias son también ocasión para rogativas y procesiones. El proceso epidémico de 1598-1600 dio lugar a numerosos actos religiosos, como la procesión general celebrada el 20 de diciembre de 1598, que recogen las actas capitulares de este año ${ }^{33}$. En ocasiones basta la posibilidad de contagio para organizar unas rogativas; en marzo de 1650 se acuerda hacer una procesión general a la ermita de San Roque por la peste que se extiende por Andalucía, que se completa con una misa rezada en la ermita y otra cantada con sermón en la catedral a la vuelta de la procesión ${ }^{34}$.

Otros sucesos particulares dan pie al cabildo para organizar funciones religiosas del tipo que comentamos. El 7 de mayo de 1729 se acuerda «que para el buen suceso de el reedificio de la torre [de la catedral], aiga una misa solemne... y que para ese día [que ha de señalar el maestro de ceremonias] se traiga el cuerpo de la gloriosa Santa Eulalia, nuestra patrona, a la capilla mayor» ${ }^{35}$.

No todas las rogativas obedecen únicamente a la iniciativa de los poderes civiles y eclesiásticos. Esto, que es más notorio en los núcleos rurales, también se pone de manifiesto en Oviedo, donde instituciones

31 ACO, libro de acuerdos n." 19, f. 406.

32 ACO, libro de acuerdos n." 21, f. 347.

33 En ACO, libro de acuerdos n." 20, fs. 192 vto.-193. Destaca en esta procesión el hecho de que sea utilizado el santo sudario, reliquia que únicamente se mostraba al pueblo tres veces al año; la relación de cómo se realizaban estas funciones se puede encontrar en Ambrosio de MORALES, Viage de... por orden del rey D. Phelipe II a los reynos de León, y Galicia y Principado de Asturias (Madrid: Imprenta de Antonio Marín, 1765) (edic. fac. Oviedo: Biblioteca Popular Asturiana, 1977), pp. 79-80. El viaje es del año 1572.

34 Ver ACO, Cabildos espirituales, libro 1, f. 46.

$35 \mathrm{ACO}$, libro de acuerdos n." 46, f. 149 vto. 
como las cofradías son las que pueden proponer y convocar. Es el caso de la cofradía de Nuestra Señora del Rosario de Oviedo, que solicita hacer una procesión con su imagen en mayo de 1664 en atención a la sequía de ese momento ${ }^{36}$.

\section{LA INFLUENCIA EN LA VIDA COTIDIANA}

Con independencia de cuál sea la institución convocante, todas las rogativas ponen en movimiento a la ciudad. Está presente y participa la «masa popular», a la que suele advertirse mediante pregón; las órdenes religiosas intervienen corporativamente en los actos mediante la celebración de misas, participación en las procesiones ${ }^{37}$, etc. También las cofradías, como tales, suelen ser convocadas a estas funciones religiosas, y a ellas asisten portando sus cruces y estandartes.

En sus recorridos, las procesiones de rogativas ocupaban las zonas más importantes: la plaza mayor y la calle del Sol ${ }^{38}$, campo de San Francisco y campo de Santa Clara ${ }^{39}$; en general, el punto de referencia para las rogativas es la plaza de la catedral, desde la cual las procesiones se dirigían hacia los conventos de la ciudad; además de los de San Francisco y Santa Clara, ya señalados, los de Santo Domingo, Santa María de la Vega y San Vicente.

La presencia de las órdenes religiosas y de las cofradías, así como la ocupación del casco urbano, pueden dar una idea aproximada de la incidencia de estas funciones religiosas en el acontecer ovetense. $\mathrm{Si}$ a esto añadimos los avisos y pregones previos hechos por el cabildo y ayuntamiento, los toques de campana para la convocatoria inmediata, los sermones y las celebraciones litúrgicas, el cuadro esquemático del desarrollo de las rogativas queda completo.

Las rogativas, dijimos al principio, aportan información sobre las devociones de más arraigo. En el caso ovetense destaca el recurso a Santa Eulalia de Mérida, patrona de la ciudad; sus reliquias suelen sacarse en

36 Ver Ciriaco Miguel Vigil, op. cit., pp. 501-502.

37 Para la procesión de rogativa acordada el 20 de marzo de 1586 (señalada en nota 28), se pide «a los relixiosos hagan proçesiones en sus casas o bengan a esta iglesia [catedral]».

38 Ver ACO, libro de acuerdos n." 38, f. 196 vto.: procesión en petición de lluvias celebrada el 14 de mayo de 1704; partió de la catedral para volver a ella.

39 Ver ibid., f. 197 vto. Procesión por el mismo motivo, q1.e se hizo el 21 de mayo. 
procesión para pedir que mejore el tiempo o se produzcan las necesarias lluvias. Otras devociones particulares de la ciudad que se ponen de manifiesto en las rogativas son las que tienen como centro a las reliquias conservadas en la Cámara Santa de la catedral - «cuerpos santos», se lee en algunos lugares-, en particular el Santo Sudario, y la Cruz de la Victoria. También destaca la devoción a Nuestra Señora del Rey Casto (en la capilla del mismo nombre en la catedral ovetense), a Nuestra Señora del Rosario en el convento de Santo Domingo, y al Cristo de la Piedad del convento de San Francisco. Estas devociones perduran durante toda la época que hemos analizado ${ }^{40}$, particularmente la de Santa Eulalia: a mediados del siglo XIX se sigue sacando en procesión en demanda de lluvias ${ }^{41}$.

\section{A MODO DE RESUMEN}

Las crisis epidémicas y de subsistencias, con la proximidad de la muerte que traían consigo y las situaciones conflictivas derivadas de hechos políticos y enfrentamientos armados generaron - como hemos vistonumerosas manifestaciones públicas de carácter religioso en Oviedo durante el Antiguo Régimen. Estas funciones tuvieron el suficiente eco corno para dar lugar a una movilización general de la ciudad, lo que puede tomarse como síntoma de preocupación general ante las causas (pıecxupación de diferente graduación, según el carácter más o menos inmediato del problema); pero también una muestra de la presencia y arraigo general de los valores religiosos.

La iniciativa y organización de las rogativas parten de instancias sociales bien determinadas, pero esto no excluye que se trate de actos que respondan a unos deseos, explícitos o implícitos, de las bases sociales. Hay que señalar, no obstante, que en los casos de rogativas por los sucesos políticos (20\%) y la familia real (20\%) tal vez el sentimiento popular no sea tan consciente y su realización haya sido más

4) El 2 de octubre de 1819 salió una procesión con las imágenes de San Roque, Santa Eulalia y Nuestra Señora del Rey Casto con motivo de la epidemia que afligía a Andalucía; la rogativa había sido solicitada por una real cédula del 22 de septiembre (ver Ciriaco Miciuel. Visill, op. cit.. p. 454).

1 El 15 de agosto de 1847 el ayuntamiento de Oviedo pide al cabildo de la catedral que saque a Santa Eulalia para que llueva lo necesario y no se pierda la cosecha (ver ACO, libro de acuerdos n.", fs. 337 y vto.). Al año siguiente, el 11 de agosto, se lee en las actas: "En atención a que aun no ha llovido lo suficiente para salvar la cosecha, se acordó continúe en rogativa nuestra Santa Patrona» (ibid., f. 365). 
el resultado de las órdenes recibidas; en cualquier caso, la instrumentalización de la práctica religiosa por la autoridad civil lo que viene a señalar es, precisamente, el arraigo de tal prácrica.

Según Isabel Lobato, las rogativas y procesiones son una muestra del dominio ideológico de la Iglesia sobre el poder civil ${ }^{42}$. Tal afirmación nos parece discutible; incluso podría formularse cambiando el orden de los términos, pues cabe preguntarse si no sería el poder civil el que se sirvió en ocasiones del prestigio y autoridad de las instituciones eclesiásticas y de las creencias religiosas de la sociedad para mantener el orden en circunstancias críticas (epidemias, hambres) o para hacer más populares ciertas actuaciones políticas. Debe tenerse en cuenta, además, que las creencias de quienes ostentan la autoridad civil son básicamente las mismas que las del conjunto social español y, por supuesto, que las que tienen los eclesiásticos; otra cosa es el modo en que tales creencias se plasman en la vida de cada sector social. Si toda la vida de los europeos durante la Edad Moderna - y en particular de los españolesse vive bajo el signo de la religión, como señala Lucien Febvre, es porque la sociedad, consciente o inconscientemente, ha asumido esos valores religiosos ${ }^{43}$; su religiosidad parte de la propia sociedad, no es algo impuesto desde fuera. Nos parece que plantear el fenómeno religioso -en particular el que aquí comentamos- en términos de poder y dominio es simplificar peligrosamente una realidad que tiene unos componentes muy diversos y con relaciones complejas.

Desde finales del XVIII y más claramente desde comienzos del XIX, se observa en Oviedo un proceso de paulatina secularización de algunos actos personales como es el otorgamiento de últimas voluntades, cuya consecuencia es la progresiva limitación de lo religioso a la esfera familiar. El proceso alcanza también a manifestaciones colectivas como es el caso de las cofradías, que dejarán a un lado cometidos sociales y de asistencia para atender sólo a fines religiosos ${ }^{44}$. Esta modificación no

42 Ver Isabel Lobato I Franco, op. cit., p. 434.

43 «Desde su nacimiento [el hombre] se hallaba inmerso en un baño de cristianismo del que ni siquiera en el momento de la muerte podía librarse»; «nosotros no somos teólogos y las gentes del siglo XVI lo eran. Incluso cuando no habían pasado años en un convento». Estas palabras de Lucien Febvre (citadas en Bartolomé BENNASSAR, Valladolid en el siglo de oro [Ayuntamiento de Valladolid, 1983], pp. 349 y 354 respectivamente), aunque se refieren al XVI, son aplicables al XVII y gran parte del XVIII español. En lo concerniente a Oviedo, sólo a finales de este siglo aparecen algunos casos muy aislados de oposición al catolicismo.

44 Ver las conclusiones de Roberto J. LÓPEZ, Oviedo: muerte y religiosidad en el siglo XVIII (Oviedo: Servicio de Publicaciones del Principado de Asturias, 1985); y Com. 
parece afectar a las rogativas. Al igual que en Barcelona ${ }^{45}$, se mantienen vigentes las plegarias públicas durante todo el siglo XVIII y hasta mediados del XIX. No podemos señalar, sin embargo, la calidad de esta vigencia; es decir, si el grado de participación de los ovetenses es el mismo con relación a los siglos anteriores, o si ha disminuido; si el convencimiento sobre su eficacia real permanece, o es una ceremonia formal. De todos modos, es indudable la influencia que la naciente mentalidad burguesa tendrá sobre estas celebraciones públicas, y que si bien no es apreciable en la primera mitad del siglo - al menos con los elementos aquí utilizados, sí lo será en años posteriores, hasta quedar relegadas las rogativas a los núcleos de población rural.

\author{
ROBERTO J. LÓPEZ \\ Facultad de Geografía e Historia \\ Universidad de Santiago
}

El estudio de las rogativas públicas pone de relieve varias cuestiones. Desde el punto de vista conceptual, el análisis de estas prácticas muestra con claridad la excesiva simplicidad de la división entre formas culturales elitistas y populares, y la necesidad de hallar modos para definir adecuadamente la pertinencia de fenómenos sociales como éste. Desde la óptica cultural y sociológica, las rogativas informan sobre los valores religiosos de una sociedad; pero también sobre sus miedos y temores cotidianos, así como sobre los intentos de los poderes públicos para lograr una mayor cohesión social en determinados momentos. Tratamos de desarrollar estos tres puntos centrándonos en la ciudad de Oviedo.

Some questions are amphatized by public prayers study. Conceptually, analysis of these practices shows the excessive simplicity of the division between elitist and popular cultural forms, and the need of finding ways to establish appropriately the pertinence of social phenomena like these. From a cultural and sociological point of view, public prayers provide information about religious valours of a society; but also on her daily fears, and attempts of authorities to acheve a social cohesion at particular moments. We try to develop these three aspects in this research into the Spanish city of Oviedo.

portamientos religiosos en Asturias durante el Antiguo Régimen (Gijón: Silverio Cañada Editor, 1989).

45 Ver Magdalena Mirabet i Cucala, op. cit., p. 493. 